\title{
A model of knowledge management system for facilitating knowledge as a service (KaaS) in cloud computing environment
}

\begin{abstract}
Knowledge as a service (KaaS) is an emerging concept that integrates knowledge management (KM), a knowledge organization, and knowledge markets. KaaS are programs that provide content-based (data, information, knowledge) as organizational outputs (e.g., advice, answers, facilitation), to meet person or external user wants or needs. KaaS are delivered through the knowledge markets as a cloud computing (CC) environment. In ensuring the services will be delivered to the right community of practice $(\mathrm{CoP})$ at the right time in a proper manner, therefore there is a need of a system called knowledge management system (KMS), so that the KaaS can be well managed in a proper form by using the KM life cycle processes. These life cycle processes are including the knowledge acquisition, knowledge storage, knowledge dissemination and knowledge application. This paper presents the concept and its model for facilitating knowledge as a service (KaaS) in a KM system so that CoP can make used the knowledge from service provider as organizational output for their referencing in the context of current best practice and lesson learnt especially related to the CC environment. By using this KMS model, the community who are engaged or connected to the cloud can be easily getting the KaaS as they are wanted or to be considered for the potential purposes in achieving their goal or mission statement.
\end{abstract}

Keyword: Knowledge as service; Knowledge management; Knowledge management system; Knowledge process; Cloud computing 